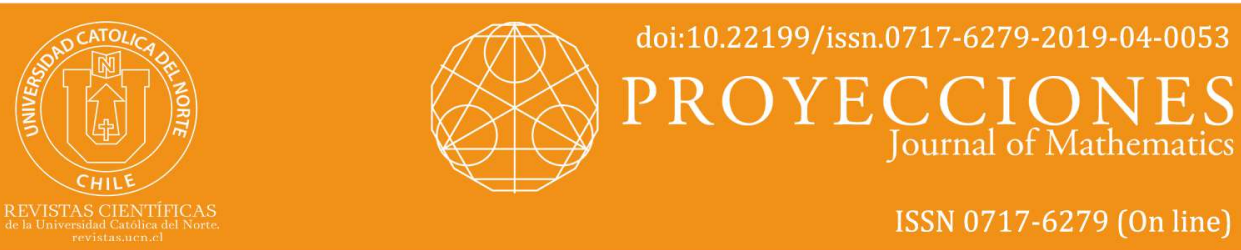

\title{
On the inverse eigenproblem for symmetric and nonsymmetric arrowhead matrices
}

\author{
H. Pickmann-Soto* iD orcid.org/0000-0002-2472-0550 \\ Susana Arela** (iD) orcid.org/0000-0003-4214-6908 \\ J. Egaña** iD orcid.org/0000-0003-0033-2120 \\ D. Carrasco Olivera ${ }^{* * *}$ (iD) orcid.org/0000-0003-2868-534X \\ *Universidad de Tarapacá, Dept. de Matemática, Arica, Chile. \\ $\nabla$ hpickmanns@academicos.uta.cl \\ **Universidad de Tarapacá, Dept. de Matemática, Arica, Chile. \\ susana.arela.p@gmail.com \\ ${ }^{* * *}$ Universidad Católica del Norte, Dept. de Matemáticas, Antofagasta, Chile \\ N jegana@ucn.cl \\ ${ }^{* * * *}$ Universidad del Bio Bio, Dept de Matemática, Concepción, Chile. \\ \dcarrasc@ubiobio.cl
}

Received: May 2018 | Accepted: June 2019

\section{Abstract:}

We present a new construction of a symmetric arrow matrix from a particular spectral information: let $\lambda^{(n)}$ be the minimal eigenvalue of the matrix and $\lambda_{j}^{(1)}, j=1,2, \ldots, n$ the maximal eigenvalues of all leading principal submatrices of the matrix. We use such a procedure to construct a nonsymmetric arrow matrix from the same spectral information plus to an eigenvector $x^{(n)}=$ $\left(x_{1}, x_{2}, \ldots, x_{n}\right)$, so that $\left(x^{(n)}, \lambda_{n}^{(n)}\right)$ is an eigenpair of the matrix. Moreover, our results generate an algorithmic procedure to compute a solution matrix.

Keywords: Arrow matrices; Symmetric and nonsymmetric matrix; Inverse eigenvalue problem.

MSC (2010): 65F15, 65F18, 15 A18.

\section{Cite this article as (IEEE citation style):}

H. Pickmann, S. Arela, J. Egaña and D. Carrasco, "On the inver-

se eigenproblem for symmetric and nonsymmetric arrowhead matrices", Proyecciones (Antofagasta, On line), vol. 38 , no. 4 , pp. 811-828, Oct. 2019 , doi: $10.22199 /$ issn.07176279-2019-04-0053. [Accessed dd-mm-yyyy].

Article copyright: (C) 2019 Hubert Pickmann Soto, Susana Arela Pérez, Juan Carlos Egaña and Dante Carrasco Olivera. This is an open access article distributed under the terms of the Creative Commons Licence, which permits unrestricted use and distribution provided the original author and source are credited.

(cc) BY 


\section{Introducction}

We consider a particular inverse eigenvalue problem for a real arrowhead matrix of the form:

$$
A=\left(\begin{array}{ccccc}
a_{1} & & & & b_{1} \\
& a_{2} & & & b_{2} \\
& & \ddots & & \vdots \\
& & & a_{n-1} & b_{n-1} \\
c_{1} & c_{2} & \cdots & c_{n-1} & a_{n}
\end{array}\right) ; b_{i} c_{i}>0, i=1,2, \ldots, n-1 .
$$

This type of matrices appear in various problems of applications in science and engineering such as wireless communications systems [12], radiationless transitions in isolated molecules [1] and eigenvalues problems [3], and it is well studied especially in the symmetrical case, that is, when $b_{i}=c_{i}, i=1,2, \ldots, n-1$. The downwards arrowhead matrices and the upwards arrowhead matrices, appear indistinctly in the literature, this is because the classical inverse eigenvalue problem for these is equivalent, since they are similar. We understand that the classical inverse eigenvalue problem, generally only considers spectral information of the entire matrix (see $[2]$ ).

In this paper we present new constructions of a downward arrowhead matrix, in the symmetrical and nonsymmetrical cases, from a particular spectral information being used recently ([4] - [8]). In the symmetric case: let $\lambda_{j}^{(j)}$ be the maximal eigenvalues of all leading submatrices of the matrix and $\lambda_{1}^{(n)}$ the minimal eigenvalue of the matrix. A very important fact is that by considering this spectral information the inverse eigenvalue problem for the downwards arrowhead matrices is not equivalent to the inverse eigenvalue problem for the arrowhead matrices upwards, since the eigenvalues of the leading principal submatrices are not invariant under some process of similarity between these matrices.

However, an interesting fact that relates the symmetrical case with the nonsymmetric case is that a arrowhead matrix of the form (1.1) is diagonally similar to an irreducible arrowhead matrix 


$$
B=\left(\begin{array}{lllll}
a_{1} & & & & d_{1} \\
& a_{2} & & & d_{2} \\
& & \ddots & & \vdots \\
& & & a_{n-1} & d_{n-1} \\
d_{1} & d_{2} & \cdots & d_{n-1} & a_{n}
\end{array}\right),
$$

where $d_{i}=\sqrt{b_{i} c_{i}} ; 1,2, \ldots, n-1$. Indeed, if we consider the matrix $D=$ $\operatorname{diag}\left(\mu_{1}, \mu_{2}, \ldots, \mu_{n}\right)$, with $\mu_{i}=\sqrt{\frac{c_{i}}{b_{i}}}, i=1,2, \ldots, n-1$ and $\mu_{n}=1$, we obtain that $D A D^{-1}=B$. As a consequence the spectral properties of a symmetric arrowhead matrix of the form (1.2) are transferred to a nonsymmetric arrowhead matrix of the form (1.1).

In the nonsymmetric case there are also some advances ([6], [11], [13]) and recently in [10] a construction of a nonsymmetric upwards arrowhead matrix is presented. In this paper, the authors consider an eigenvector $\mathbf{x}^{(n)}=\left(x_{1}, x_{2}, \ldots, x_{n}\right)$, associated with the maximal eigenvalue of the matrix, as spectral information additional to that one considered in the symmetric case [9]. As far as we know, the cases of constructing an arrowhead matrix of the form (1.2) or (1.1), from the spectral information that we use, has not been considered in the literature. Motivated by everything mentioned above we consider the following problems.:

Problem 1. Given the list of real numbers $\left\{\lambda_{1}^{(n)}\right\} \cup\left\{\lambda_{j}^{(j)}\right\}_{j=1}^{n}$, construct a matrix $B$ of the form (1.2), such that $\lambda_{1}^{(n)}$ is the minimal eigenvalue of $B$, and $\lambda_{j}^{(j)}$ are the maximal eigenvalues of the leading principal submatrix $B_{j}$, $j=1,2, \ldots, n$ of $B$.

Problem 2. Given the list of real numbers $\left\{\lambda_{1}^{(n)}\right\} \cup\left\{\lambda_{j}^{(j)}\right\}_{j=1}^{n}$ and the vector $\mathbf{x}^{(n)}=\left(x_{1}, x_{2}, \ldots, x_{n}\right)$, construct a matrix $A$ of the form (1.1), such that $\lambda_{1}^{(n)}$ is the minimal eigenvalue of $A$, and $\lambda_{j}^{(j)}$ are the maximal eigenvalue of the leading principal submatrix $A_{j}, j=1,2, \ldots, n$ of $A$, and $\left(\mathbf{x}^{(n)}, \lambda_{n}^{(n)}\right)$ is an eigenpair of $A$.

\section{Solution to Problem 1}

We start this section by recalling the following lemmas: 
Lemma 1. [9] Let $P(\lambda)$ be a monic polynomial of degree $n$ with all zeroes being real. If $\lambda_{1}$ and $\lambda_{n}$ are, respectively, the minimal and maximal zero of $P(\lambda)$, then

1. If $\mu<\lambda_{1}$, we have $(-1)^{n} P(\mu)>0$,

2. If $\mu>\lambda_{n}$, we have $P(\mu)>0$.

Lemma 2. Let $B$ be an $n \times n$ symmetric arrowhead matrix of the form (1.2) with $d_{i}>0, i=1,2, \ldots, n-1$, and let $B_{j}$ be the $j \times j$ leading principal submatrix of $B$, with characteristic polynomial $P_{j}(\lambda)=\operatorname{det}\left(\lambda I_{j}-B_{j}\right)$, $j=1,2, \ldots, n$. Then the sequence $\left\{P_{j}(\lambda)\right\}_{j=1}^{n}$ satisfies the recurrence relation

$$
\begin{gathered}
P_{j}(\lambda)=\prod_{i=1}^{j-1}\left(\lambda-a_{i}\right) ; \quad j=1,2, \ldots, n-1 . \\
P_{j}(\lambda)=\left(\lambda-a_{j}\right) P_{j-1}(\lambda)-\sum_{k=1}^{j-1} d_{k}^{2} \prod_{i=1, i \neq k}^{j-1}\left(\lambda-a_{i}\right) ; \quad j=n .
\end{gathered}
$$

Observe that from the Cauchy interlacing property, the minimal and the maximal eigenvalue, $\lambda_{1}^{(j)}$ and $\lambda_{j}^{(j)}$ respectively, of each leading principal submatrix $B_{j}, j=1,2, \ldots, n$, of irreducible matrix $B$ in (1.2) satisfy the relations:

$$
\lambda_{1}^{(n)}<\lambda_{1}^{(n-1)} \leq \cdots \leq \lambda_{1}^{(2)} \leq \lambda_{1}^{(1)} \leq \lambda_{2}^{(2)} \leq \cdots \leq \lambda_{n-1}^{(n-1)}<\lambda_{n}^{(n)}
$$

and

$$
\lambda_{1}^{(n)}<a_{i}<\lambda_{n}^{(n)} ; i=1,2, \ldots, n .
$$

Theorem 1. Let $\left\{\lambda_{1}^{(n)}\right\} \cup\left\{\lambda_{j}^{(j)}\right\}_{j=1}^{n}$ be the real numbers satisfying

$$
\lambda_{1}^{(n)}<\lambda_{1}^{(1)}<\lambda_{2}^{(2)}<\lambda_{3}^{(3)} \cdots<\lambda_{n-1}^{(n-1)}<\lambda_{n}^{(n)} .
$$

Then, there exists an arrowhead symmetric matrix $B$, of the form (1.2), of order $n$, such that $\lambda_{1}^{(n)}$ is the minimal eigenvalue of de matrix $B$ and $\lambda_{j}^{(j)}$ is the maximal eigenvalue of the leading principal submatrix $B_{j}, j=$ $1,2, \ldots, n$ of the matrix $B$. 
Proof. Suppose that $\left\{\lambda_{1}^{(n)}\right\} \cup\left\{\lambda_{j}^{(j)}\right\}_{j=1}^{n}$ satisfies (2.4). To show the existence of a symmetric arrowhead matrix $\mathrm{B}$ with the required properties is equivalent to show that the system of equations

$$
\begin{aligned}
P_{n}\left(\lambda_{1}^{(n)}\right) & =0, \\
P_{j}\left(\lambda_{j}^{(j)}\right) & =0 ; \quad j=1,2, \ldots, n
\end{aligned}
$$

where $P_{j}(\lambda)=\operatorname{det}\left(\lambda I_{j}-B_{j}\right), j=1,2, \ldots, n$ satisfies Lemma 2 , has real solutions $a_{j}, j=1,2, \ldots, n$ and $d_{i}>0, i=1, \ldots, n-1$.

The previous system can be written as:

$$
\begin{gathered}
P_{n}\left(\lambda_{1}^{(n)}\right)=\left(\lambda_{1}^{(n)}-a_{n}\right) P_{n-1}\left(\lambda_{1}^{(n)}\right)-\sum_{k=1}^{n-1} d_{k}^{2} \prod_{i=1, i \neq k}^{n-1}\left(\lambda_{1}^{(n)}-a_{i}\right)=0 \\
P_{j}\left(\lambda_{j}^{(j)}\right)=\prod_{i=1}^{j}\left(\lambda_{j}^{(j)}-a_{i}\right)=0 ; \quad j=1,2, \ldots, n-1 . \\
P_{n}\left(\lambda_{n}^{(n)}\right)=\left(\lambda_{n}^{(n)}-a_{n}\right) P_{n-1}\left(\lambda_{n}^{(n)}\right)-\sum_{k=1}^{n-1} d_{k}^{2} \prod_{i=1, i \neq k}^{n-1}\left(\lambda_{n}^{(n)}-a_{i}\right)=0 .
\end{gathered}
$$

From (2.5) for $j=1,2, \ldots, n-1$ it follows that

$$
a_{j}=\lambda_{j}^{j} ; \quad j=1,2, \ldots, n-1 .
$$

Now, from Lemma (1) and condition (2.4) we have that $P_{n-1}\left(\lambda_{1}^{(n)}\right) \neq 0$ and $P_{n-1}\left(\lambda_{n}^{(n)}\right) \neq 0$, so

$$
\begin{gathered}
a_{n}=\lambda_{1}^{(n)}-\frac{1}{P_{n-1}\left(\lambda_{1}^{(n)}\right)} \sum_{k=1}^{n-1} d_{k}^{2} \prod_{i=1, i \neq k}^{n-1}\left(\lambda_{1}^{(n)}-a_{i}\right) \\
a_{n}=\lambda_{n}^{(n)}-\frac{1}{P_{n-1}\left(\lambda_{n}^{(n)}\right)} \sum_{k=1}^{n-1} d_{k}^{2} \prod_{i=1, i \neq k}^{n-1}\left(\lambda_{n}^{(n)}-a_{i}\right) .
\end{gathered}
$$

Then,

$$
\frac{1}{P_{n-1}\left(\lambda_{n}^{(n)}\right)} \sum_{k=1}^{n-1} d_{k}^{2} \prod_{i=1, i \neq k}^{n-1}\left(\lambda_{n}^{(n)}-a_{i}\right)-\frac{1}{P_{n-1}\left(\lambda_{1}^{(n)}\right)}
$$




$$
\sum_{k=1}^{n-1} d_{k}^{2} \prod_{i=1, i \neq k}^{n-1}\left(\lambda_{1}^{(n)}-a_{i}\right)=\lambda_{n}^{(n)}-\lambda_{1}^{(n)} .
$$

Now, as $\lambda_{n}^{(n)}-\lambda_{1}^{(n)} \neq 0$, it follows that

$$
\begin{aligned}
\sum_{k=1}^{n-1} d_{k}^{2} & {\left[\frac { 1 } { ( \lambda _ { n } ^ { ( n ) } - \lambda _ { 1 } ^ { ( n ) } ) } \left(\frac{1}{P_{n-1}\left(\lambda_{n}^{(n)}\right)} \prod_{i=1, i \neq k}^{n-1}\left(\lambda_{n}^{(n)}-a_{i}\right)\right.\right.} \\
& \left.\left.-\frac{1}{P_{n-1}\left(\lambda_{1}^{(n)}\right)} \prod_{i=1, i \neq k}^{n-1}\left(\lambda_{1}^{(n)}-a_{i}\right)\right)\right]=1,
\end{aligned}
$$

This last equation can be written in the form:

$$
\sum_{k=1}^{n-1} D_{k}=1
$$

where

$$
\begin{aligned}
D_{k}=d_{k}^{2} & {\left[\frac { 1 } { ( \lambda _ { n } ^ { ( n ) } - \lambda _ { 1 } ^ { ( n ) } ) } \left(\frac{1}{P_{n-1}\left(\lambda_{n}^{(n)}\right)} \prod_{i=1, i \neq k}^{n-1}\left(\lambda_{n}^{(n)}-a_{i}\right)\right.\right.} \\
& \left.\left.-\frac{1}{P_{n-1}\left(\lambda_{1}^{(n)}\right)} \prod_{i=1, i \neq k}^{n-1}\left(\lambda_{1}^{(n)}-a_{i}\right)\right)\right] .
\end{aligned}
$$

From (2.3) and the condition (2.4) we set the expression

$$
\begin{gathered}
\frac{1}{P_{n-1}\left(\lambda_{n}^{(n)}\right)} \prod_{i=1, i \neq k}^{n-1}\left(\lambda_{n}^{(n)}-a_{i}\right)-\frac{1}{P_{n-1}\left(\lambda_{1}^{(n)}\right)} \\
\prod_{i=1, i \neq k}^{n-1}\left(\lambda_{1}^{(n)}-a_{i}\right)>0,
\end{gathered}
$$

for each $k=1,2, \ldots, n-1$.

Now, considering the particular solution of (2.7)

$$
D_{1}=D_{2}=\cdots=D_{n-1}=\frac{1}{n-1},
$$

we obtain that 


$$
d_{k}=\sqrt{\frac{\left(\lambda_{n}^{(n)}-\lambda_{1}^{(n)}\right)}{(n-1)\left(\frac{1}{P_{n-1}\left(\lambda_{n}^{(n)}\right)} \prod_{i=1, i \neq k}^{n-1}\left(\lambda_{n}^{(n)}-a_{i}\right)-\frac{1}{P_{n-1}\left(\lambda_{1}^{(n)}\right)} \prod_{i=1, i \neq k}^{n-1}\left(\lambda_{1}^{(n)}-a_{i}\right)\right)}}
$$

for $k=1,2, \ldots, n-1$. Finally, by replacing the values of $d_{k}$ in $(2.7)$, we obtain the value of $a_{n}$.

An immediate consequence of this result is that the matrix $B$ with the required spectral properties is not unique. In the proof of the previous theorem we guarantee its existence and only provide a particular solution.

An interesting situation of the similarity indicated above, is that the eigenvalues of all the leading submatrices are invariant. In this sense, from the data considered in the previous theorem we can construct a nonsymmetric arrowhead matrix $A$ of the form (1.1). That is, we first construct a symmetric arrowhead matrix $B$ of the form (1.2), then we use the similarity process indicated above to determine the entries $b_{i}$ and $c_{i}$ through the solutions of the equations $d_{i}=\sqrt{b_{i} c_{i}} ; 1,2, \ldots, n-1$.

\section{Solution to Problem 2}

In this section we construct a nonsymmetric arrowhead matrix $A$ of the form (1.1) without going through the similarity process mentioned above. It should be noted that the construction in the following theorem gives us uniqueness in the entries $a_{i}$ and $b_{i}$ for $i=1,2, \ldots, n-1$, and a particular solution can by choose for $c_{i}$. In contrast, the construction via similarity gives us only uniqueness for the diagonal entries $a_{i}$.

Lemma 3. [7] Let $\mathbf{x}_{1}, \mathbf{x}_{2}, \ldots, \mathbf{x}_{n}$ be a set of orthonormal eigenvectors associated to the eigenvalues $\lambda_{1}, \lambda_{2}, \ldots, \lambda_{n}$ of an $n \times n$ matrix $B$ of the form (1.2), with all its diagonal entries $a_{j}$ distinct, $j=2,3, \ldots, n$, and all its entries $b_{i}$ positive, $i=1,2, \ldots, n-1$. Then $x_{\mu j} \neq 0$ for $\mu, j=1,2, \ldots, n$, where $x_{\mu j}$ denotes the $\mu-$ th entry of the vector $\mathbf{x}_{j}$.

Lemma 4. Let $A$ be an $n \times n$ nonsymmetric arrow matrix of the form (1.1), and let $A_{j}$ be the $j \times j$ leading principal submatrix of $A$, with characteristic 
polynomial $P_{j}(\lambda)=\operatorname{det}\left(\lambda I_{j}-A_{j}\right), j=1,2, \ldots, n$. Then the sequence $\left\{P_{j}(\lambda)\right\}_{j=1}^{n}$ satisfies the recurrence relation

$$
\begin{gathered}
P_{j}(\lambda)=\prod_{i=1}^{j}\left(\lambda-a_{i}\right) \quad j=1,2, \ldots, n-1 . \\
P_{j}(\lambda)=\left(\lambda-a_{j}\right) P_{j-1}(\lambda)-\sum_{k=1}^{j-1} b_{k} c_{k} \prod_{\substack{i=1 \\
i \neq k}}^{j-1}\left(\lambda-a_{i} ; j=n .\right.
\end{gathered}
$$

To give a solution to Problem (2), that is, to build an $n \times n$ nonsymmetric arrowhead matrix of the form (1.1) we present the following result:

Theorem 1. Let the real numbers $\left\{\lambda_{1}^{(n)}\right\} \cup\left\{\lambda_{j}^{(j)},\right\}_{j=1}^{n}$ and the vector $\mathbf{x}^{(n)}=$ $\left(x_{1}, x_{2}, \ldots, x_{n}\right)$ that

$$
\lambda_{1}^{(n)}<\lambda_{1}^{(1)}<\lambda_{2}^{(2)}<\lambda_{3}^{(3)} \cdots<\lambda_{n-1}^{(n-1)}<\lambda_{n}^{(n)}
$$

and

$$
x_{i} x_{i+1}>0, i=1,2, \ldots, n-1 .
$$

Then, there exists an $n \times n$ nonsymmetric downward arrowhead matrix $A$ of the form (1.1), such that $\lambda_{1}^{(n)}$ is the minimal eigenvalue of the matrix $A, \lambda_{j}^{(j)}, j=1,2, \ldots, n$ the maximal eigenvalues of all leading principal submatrices of the matrix $A$ and $\left(x^{(n)}, \lambda_{n}^{(n)}\right)$ an eigenpair of $A$.

Proof. To show the existence of a nonsymmetric arrowhead-down ma$\operatorname{trix} A$ with the required properties is equivalent to show that the system of equations

$$
\begin{gathered}
P_{j}\left(\lambda_{j}^{(j)}\right)=0, j=1,2, \ldots, n-1 \\
P_{n}\left(\lambda_{j}^{(n)}\right)=0, j=1, n \\
A \mathbf{x}^{(n)}=\lambda_{n}^{(n)} \mathbf{x}^{(n)},
\end{gathered}
$$

where $P_{j}(\lambda)=\operatorname{det}\left(\lambda I_{j}-A_{j}\right), j=1,2, \ldots, n$ satisfies Lemma 4 , has real solutions $a_{j}, j=1,2, \ldots, n$ and $b_{i}, c_{i}, i=1,2, \ldots, n-1$, with $c_{i} b_{i}>0$.

From Lemma 4 the system (2.11) can be written as 


$$
\begin{gathered}
P_{j}\left(\lambda_{j}^{j}\right)=\prod_{i=1}^{j}\left(\lambda_{1}^{j}-a_{i}\right)=0, \quad j=1,2, \ldots, n-1 ; \\
P_{n}\left(\lambda_{1}^{n}\right)=\left(\lambda_{1}^{n}-a_{n}\right) P_{n-1}\left(\lambda_{1}^{n}\right)-\sum_{k=1}^{n-1} b_{k} c_{k} \prod_{\substack{i=1 \\
i \neq k}}^{j-1}\left(\lambda_{1}^{n}-a_{i}\right)=0, \\
P_{n}\left(\lambda_{n}^{n}\right)=\left(\lambda_{n}^{n}-a_{n}\right) P_{n-1}\left(\lambda_{n}^{n}\right)-\sum_{k=1}^{n-1} b_{k} c_{k} \prod_{\substack{i=1 \\
i \neq k}}^{j-1}\left(\lambda_{1}^{n}-a_{i}\right)=0, \\
a_{j} x_{j}+b_{j} x_{n}=\lambda_{n}^{(n)} x_{j}, j=1,2,3, \ldots, n-1 \\
\sum_{k=1}^{n-1} c_{k} x_{k}+a_{n} x_{n}=\lambda_{n}^{(n)} x_{n} .
\end{gathered}
$$

From (2.12) it follows that

$$
a_{j}=\lambda_{j}^{j} ; \quad j=1,2, \ldots, n-1 .
$$

Then from (2.13) and condition (3.3) we have

$$
b_{j}=\left(\lambda_{n}^{(n)}-a_{j}\right) \frac{x_{j}}{x_{n}} ; \quad j=1,2, \ldots, n-1 .
$$

From (2.12), condition (3.2) and Lemma 1, for $j=n$ we have

$$
\begin{gathered}
a_{n}=\lambda_{1}^{(n)}-\frac{1}{P_{n-1}\left(\lambda_{1}^{(n)}\right)} \sum_{k=1}^{n-1} b_{k} c_{k} \prod_{i=1}^{i \neq k} b_{k} c_{k} \prod_{\substack{i=1 \\
i \neq k}}^{j-1}\left(\lambda_{1}^{n}-a_{i}\right) \\
a_{n}=\lambda_{n}^{(n)}-\frac{1}{P_{n-1}\left(\lambda_{n}^{(n)}\right)} \prod_{k=1}^{j-1} b_{k} c_{k} \prod_{\substack{i=1 \\
i \neq k}}^{j-1}\left(\lambda_{n}^{(n)}-a_{i}\right) .
\end{gathered}
$$

Then

$$
\begin{gathered}
\sum_{k=1}^{n-1} \mathrm{~b}_{k} \mathrm{c}_{k}\left[\frac{1}{\mathrm{P}_{n-1}\left(\lambda_{1}^{(n)}\right)} \prod_{k=1}^{j-1} b_{k} c_{k} \prod_{\substack{i=1 \\
i \neq k}}^{j-1}\left(\lambda_{1}^{n}-\mathrm{a}_{i}\right)\right. \\
\left.-\frac{1}{\mathrm{P}_{n-1}\left(\lambda_{n}^{(n)}\right)} \prod_{k=1}^{j-1} b_{k} c_{k} \prod_{\substack{i=1 \\
i \neq k}}^{j-1}\left(\lambda_{n}^{(n)}-\mathrm{a}_{i}\right)\right]=\lambda_{1}^{(n)}-\lambda_{n}^{(n)}
\end{gathered}
$$


Since $\left(\lambda_{n}^{(n)}-\lambda_{1}^{(n)}\right) \neq 0$, then if we set

$$
\begin{aligned}
B_{k}=\mathrm{b}_{k} \mathrm{c}_{k} & {\left[\frac { 1 } { ( \lambda _ { n } ^ { ( n ) } - \lambda _ { 1 } ^ { ( n ) } ) } \left(\frac{1}{\mathrm{P}_{n-1}\left(\lambda_{n}^{(n)}\right)} \prod_{k=1}^{j-1} b_{k} c_{k} \prod_{\substack{i=1 \\
i \neq k}}^{j-1}\left(\lambda_{n}^{(n)}-\mathrm{a}_{i}\right)\right.\right.} \\
& -\frac{1}{\mathrm{P}_{n-1}\left(\lambda_{1}^{(n)}\right)} \prod_{k=1}^{j-1} b_{k} c_{k} \prod_{\substack{i=1 \\
i \neq k}}^{j-1}\left(\lambda_{1}^{(n)}-\mathrm{a}_{i}\right)
\end{aligned}
$$

(2.11) can be written as

$$
\sum_{k=1}^{n-1} B_{k}=1
$$

Now, by considering the particular solution

$$
B_{1}=B_{2}=B_{3} \cdots=B_{n-1}=\frac{1}{n-1}
$$

it follows that

$$
\left.c_{k}=\frac{\left(\lambda_{n}^{(n)}-\lambda_{1}^{(n)}\right)}{(\mathrm{n}-1) \mathrm{b}_{k}\left(\frac{1}{\mathrm{P}_{n-1}\left(\lambda_{n}^{(n)}\right)} \prod_{\substack{i=1 \\ i \neq k}}^{n-1}\left(\lambda_{n}^{(n)}-\mathrm{a}_{i}\right)-\frac{1}{\mathrm{P}_{n-1}\left(\lambda_{1}^{(n)}\right)} \prod_{\substack{i=1 \\ i \neq k}}^{n-1}\left(\lambda_{1}^{(n)}-\mathrm{a}_{i}\right)\right.}\right),
$$

for $k=1,2, \ldots, n-1$. Besides, from Lemma 1 and condition (3.2) we have

$$
\left(\lambda_{n}^{(n)}-\lambda_{1}^{(n)}\right)>0, \quad \frac{1}{P_{j-1}\left(\lambda_{j}^{(j)}\right)} \prod_{\substack{i=1 \\ i \neq k}}^{j-1}\left(\lambda_{j}^{(j)}-a_{i}\right)>0
$$

and

$$
-\frac{1}{P_{n-1}\left(\lambda_{1}^{(n)}\right)} \prod_{\substack{i=1 \\ i \neq k}}^{n-1}\left(\lambda_{1}^{(n)}-a_{i}\right)=-\frac{(-1)}{(-1)^{n-1} P_{j-1}\left(\lambda_{1}^{(j)}\right)} \prod_{\substack{i=1 \\ i \neq k}}^{n-1}\left(a_{i}-\lambda_{1}^{(j)}\right)>0 .
$$

Then $b_{k} c_{k}>0, k=1,2, \ldots, n-1$. Finally, by setting (3.12) and (3.10) in (3) we obtain the value of $a_{n}$. 
An important fact to note is that by following the proof of the previous theorem, we can construct a symmetric arrow matrix $B$ of the form (1.2), the most outstanding is that this construction is that it is unique and the entries of the matrix can be obtained by formulas similar to those obtained in the Theorem 1 as shown in the following corollary:

Corollary 1. Let the real numbers $\left\{\lambda_{j}^{(j)}\right\}_{j=1}^{n}$ be and let the vector $\mathbf{x}^{(n)}=$ $\left(x_{1}, x_{2}, \ldots, x_{n}\right)$ such that

$$
\lambda_{1}^{(1)}<\lambda_{2}^{(2)}<\cdots<\lambda_{n-1}^{(n-1)}<\lambda_{n}^{(n)}
$$

and

$$
x_{i} x_{i+1}>0, i=1,2, \ldots, n-1 .
$$

Then, there exists an unique $n \times n$ symmetric arrowhead matrix $B$ of the form (1.2), such that $\lambda_{j}^{(j)}$ is the maximal eigenvalue of the leading principal submatrix $B_{j}, j=1,2, \ldots, n$ of the matrix $A$ and $\left(x^{(n)}, \lambda_{n}^{(n)}\right)$ is an eigenpair of $A$.

Proof. Assuming that $b_{i}=c_{i}=d_{i}$, the expressions (3.7), (3.8), (3.9) and (3.10) in the proof of Theorem 1 become:

$$
\begin{gathered}
a_{j}=\lambda_{j}^{(j)} ; \quad j=1, \ldots, n-1 . \\
d_{j}=\left(\lambda_{n}^{(n)}-a_{j}\right) \frac{x_{j}}{x_{n}} ; \quad j=1, \ldots, n-1 . \\
a_{n}=\lambda_{n}^{(n)}-\sum_{k=1}^{n-1} d_{k} \frac{x_{k}}{x_{n}}
\end{gathered}
$$

or

$$
a_{n}=\lambda_{n}^{(n)}-\frac{1}{P_{n-1}\left(\lambda_{n}^{(n)}\right)} \sum_{k=1}^{n-1} d_{k}^{2} \prod_{i=1, i \neq k}^{n-1}\left(\lambda_{n}^{(n)}-a_{i}\right) .
$$

Another very particular, but interesting case, is when $a_{i}=a, b_{i}=b$ and $c_{i}=c$. In this case, an additional necessary condition for the eigenvector $\mathbf{x}^{(n)}=\left(x_{1}, x_{2}, \ldots, x_{n}\right)$ is that $x_{1}=x_{i}, \forall i=1,2, \ldots, n-1$. Besides, the entries of the matrix can be computed by:

$$
a=\lambda_{1}^{(1)},
$$




$$
b=\left(\lambda_{n}^{(n)}-a\right) \frac{x_{1}}{x_{n}}
$$

and

$$
c=\frac{\left(\lambda_{n}^{(n)}-a\right) x_{n}}{(n-1) x_{1}}
$$

\section{Numerical examples}

Although our results are mainly theoretical, our numerical experiments indicate some stability in the algorithms that are derived from these, when the matrix is small to medium in size. This is evidenced through the following examples.

Example 1. Given that the real numbers

$$
\begin{array}{cccccccc}
\lambda_{1}^{(7)} & \lambda_{1}^{(1)} & \lambda_{2}^{(2)} & \lambda_{3}^{(3)} & \lambda_{4}^{(4)} & \lambda_{5}^{(5)} & \lambda_{6}^{(6)} & \lambda_{7}^{(7)} \\
-10.2464 & -3.4412 & -2.3618 & -0.9103 & 0.8816 & 2.1807 & 2.6652 & 8.2581
\end{array}
$$

satisfy the condition of Theorem 1, we may construct a symmetric arrowhead matrix

$$
B=\left(\begin{array}{ccccccc}
-3.4412 & & & & & & 3.6427 \\
& -2.3618 & & & & & 3.7357 \\
& & -0.9103 & & & & 3.7771 \\
& & & 0.8816 & & & 3.6988 \\
& & & & 2.1807 & & 3.5479 \\
3.6427 & 3.7357 & 3.7771 & 3.6988 & 3.5479 & 3.4692 & -1.8239
\end{array}\right),
$$

with the required spectral properties, where

$$
\begin{aligned}
& \sigma\left(B_{1}\right)=\{-3.4412\} \text {, } \\
& \sigma\left(B_{2}\right)=\left\{\begin{array}{ll}
-3.4412 & \mathbf{- 2 . 3 6 1 8}
\end{array}\right\}, \\
& \sigma\left(B_{3}\right)=\left\{\begin{array}{lll}
-3.4412 & -2.3618 & -\mathbf{0 . 9 1 0 3}
\end{array}\right\}, \\
& \sigma\left(B_{4}\right)=\left\{\begin{array}{llll}
-3.4412 & -2.3618 & -0.9103 & \mathbf{0 . 8 8 1 6}
\end{array}\right\}, \\
& \sigma\left(B_{5}\right)=\left\{\begin{array}{lllll}
-3.4412 & -2.3618 & -0.9103 & 0.8816 & \mathbf{2 . 1 8 0 7}
\end{array}\right\} \text {, }
\end{aligned}
$$




$$
\begin{aligned}
& \sigma\left(B_{6}\right)=\left\{\begin{array}{lllllll}
-3.4412 & -2.3618 & -0.9103 & 0.8816 & 2.1807 & \mathbf{2 . 6 6 5 2}
\end{array}\right\}, \\
& \sigma\left(B_{7}\right)=\left\{\begin{array}{lllllll}
\mathbf{- 1 0 . 2 4 6 4} & -3.0483 & -1.7123 & 0.0501 & 1.5287 & 2.4604 & \mathbf{8 . 2 5 8 1}
\end{array}\right\}
\end{aligned}
$$

Example 2. Given that the real numbers

$$
\begin{array}{cccccc}
\lambda_{1}^{(1)} & \lambda_{2}^{(2)} & \lambda_{3}^{(3)} & \lambda_{4}^{(4)} & \lambda_{5}^{(5)} & \lambda_{6}^{(6)} \\
-3.2672 & -0.6768 & 0.0977 & 1.6576 & 3.3521 & 14.2928
\end{array}
$$

and the vector

$$
\mathbf{x}=\left[\begin{array}{llllll}
0.3285 & 0.0214 & -0.3925 & -0.2194 & -0.3624 & 0.7470
\end{array}\right]^{T},
$$

satisfy the conditions of Corollary 1, we construct the symmetric arrowhead matrix

$$
B=\left(\begin{array}{cccccc}
-3.2672 & & & & & 7.7211 \\
& -0.6768 & & & & 0.4297 \\
& & 0.0977 & & & -7.4580 \\
& & & 1.6576 & & -3.7115 \\
7.7211 & 0.4297 & -7.4580 & -3.7115 & -5.3079 & 3.3018
\end{array}\right),
$$

with the required spectral properties, where

$\sigma\left(B_{1}\right)=\{-3.2672\}$,

$\sigma\left(B_{2}\right)=\left\{\begin{array}{ll}-3.2672 & -\mathbf{0 . 6 7 6 8}\end{array}\right\}$,

$\sigma\left(B_{3}\right)=\left\{\begin{array}{lll}-3.2672 & -0.6768 & \mathbf{0 . 0 9 7 7}\end{array}\right\}$,

$\sigma\left(B_{4}\right)=\left\{\begin{array}{llll}-3.2672 & -0.6768 & 0.0977 & \mathbf{1 . 6 5 7 6}\end{array}\right\}$,

$\sigma\left(B_{5}\right)=\left\{\begin{array}{lllll}-3.2672 & -0.6768 & 0.0977 & 1.6576 & \mathbf{3 . 3 5 2 1}\end{array}\right\}$,

$\sigma\left(B_{6}\right)=\left\{\begin{array}{llllll}-11.5788 & -1.6499 & -0.6736 & 1.3526 & 2.7221 & \mathbf{1 4 . 2 9 2 8}\end{array}\right\}$, and $B \mathbf{x}=(14.2928) \mathbf{x}$.

Example 3. The real numbers

$$
\begin{array}{cccccccc}
\lambda_{1}^{(7)} & \lambda_{1}^{(1)} & \lambda_{2}^{(2)} & \lambda_{3}^{(3)} & \lambda_{4}^{(4)} & \lambda_{5}^{(5)} & \lambda_{6}^{(6)} & \lambda_{7}^{(7)} \\
-14.2560 & -9.0875 & -1.5235 & -1.3710 & -0.9617 & 0.0374 & 3.7273 & 4.1478
\end{array}
$$

and the real vector $\mathbf{x}=\left[\begin{array}{lllllll}0.1493 & 0.1307 & 0.0111 & -0.0756 & -0.8058 & 0.4255 & 0.3527\end{array}\right]^{T}$, satisfy the conditions of Theorem 1 . We construct a nonsymmetric arrowhead matrix 


$$
A=\left(\begin{array}{ccccccc}
-9.0875 & & & & & & 5.6024 \\
& -1.5235 & & & & & 2.1019 \\
& & -1.3710 & & & & 0.1740 \\
& & & -0.9617 & & & -1.0947 \\
& & & & 0.0374 & & -9.3903 \\
2.0350 & 5.7257 & 68.0999 & -10.3418 & -1.0428 & 2.4846 & -8.5783
\end{array}\right)
$$

with the required spectral properties, where

$$
\begin{aligned}
& \sigma\left(A_{1}\right)=\{-9.0875\}, \\
& \sigma\left(A_{2}\right)=\left\{\begin{array}{ll}
-9.0875 & \mathbf{- 1 . 5 2 3 5}
\end{array}\right\}, \\
& \sigma\left(A_{3}\right)=\left\{\begin{array}{lll}
-9.0875 & -1.5235 & -\mathbf{1 . 3 7 1 0}
\end{array}\right\}, \\
& \sigma\left(A_{4}\right)=\left\{\begin{array}{llll}
-9.0875 & -1.5235 & -1.3710 & \mathbf{- 0 . 9 6 1 7}
\end{array}\right\}, \\
& \sigma\left(A_{5}\right)=\left\{\begin{array}{lllll}
-9.0875 & -1.5235 & -1.3710 & -0.9617 & \mathbf{0 . 0 3 7 4}
\end{array}\right\}, \\
& \sigma\left(A_{6}\right)=\left\{\begin{array}{llllll}
-9.0875 & -1.5235 & -1.3710 & -0.9617 & 0.0374 & \mathbf{3 . 7 2 7 3}
\end{array}\right\}, \\
& \sigma\left(A_{7}\right)=\left\{\begin{array}{lllllll}
-\mathbf{1 4 . 2 5 6 0} & -7.6402 & -1.4552 & -1.1332 & -0.2874 & 2.8668 & \mathbf{4 . 1 4 7 8}
\end{array}\right\}, \\
& \text { and } A \mathrm{x}=(4.1478) \mathbf{x} \text {. }
\end{aligned}
$$

Example 4. We compute, for different values of $n$, a symmetric arrowhead matrix $\widetilde{B}$ from the $(n+1)$-dimensional vector $\lambda$, whose entries are real numbers chosen randomly and satisfying condition (2.4) of the Theorem 1. Figure 1 shows the plot of $e_{\lambda}=\log \left(\frac{\|\lambda-\widetilde{\lambda}\|}{\|\lambda\|}\right)$, where $e_{\lambda}$ corresponds to the logarithm of the relative error in computing $\tilde{\lambda}$, with the entries $\widetilde{\lambda}_{1}^{(n)}$, the minimal eigenvalue of de matrix $\widetilde{B}$, and $\widetilde{\lambda}_{j}^{(j)}$ the maximal eigenvalues of the $j \times j$ leading principal submatrix $\widetilde{B_{j}}$ of $\widetilde{B}, j=1,2, \ldots, n$. 


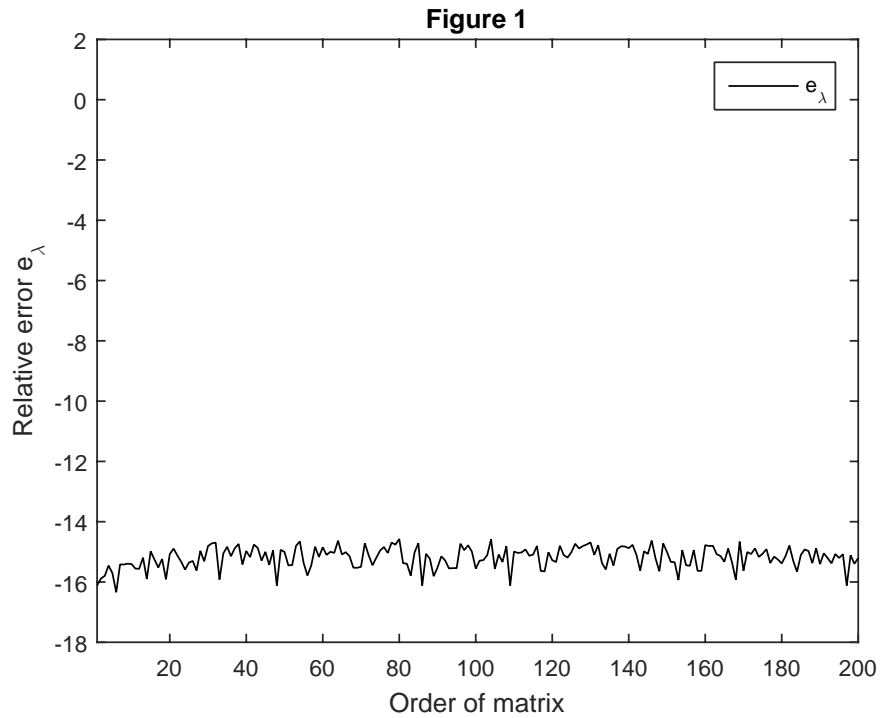

Example 5. Consider the (n)-dimensional vector $\lambda$, whose entries are real numbers chosen randomly in nondecreasing order and an $n$-dimensional vector $\mathbf{x}$, satisfying the conditions (3.13) and (3.14) of Corollary 1, respectively. From $\lambda$ and $\mathbf{x}$, we construct a symmetric arrowhead matrix $\widetilde{B}$ of order $n \times n$, such that $\widetilde{\lambda}_{j}^{(j)}$ is the maximal eigenvalue of the $j \times j$ leading principal submatrix $\widetilde{B_{j}}$ of $\widetilde{B}, j=1,2, \ldots, n$, and $\left(\widetilde{x}, \lambda_{n}^{(n)}\right)$ is an eigenpair of $\widetilde{B}$. Figure 2 shows the plot of $e_{\lambda}$, defined in example 4 , and $e_{x}=\log \left(\frac{\left\|\widetilde{B} x-\widetilde{\lambda}_{n}^{(n)} x\right\|}{\left\|\lambda_{n}^{(n)} x\right\|}\right)$ which is related to the relative error in the calculation of $\widetilde{x}$, with $n=200$ and 100 constructions of the matrix $\widetilde{B}$. 


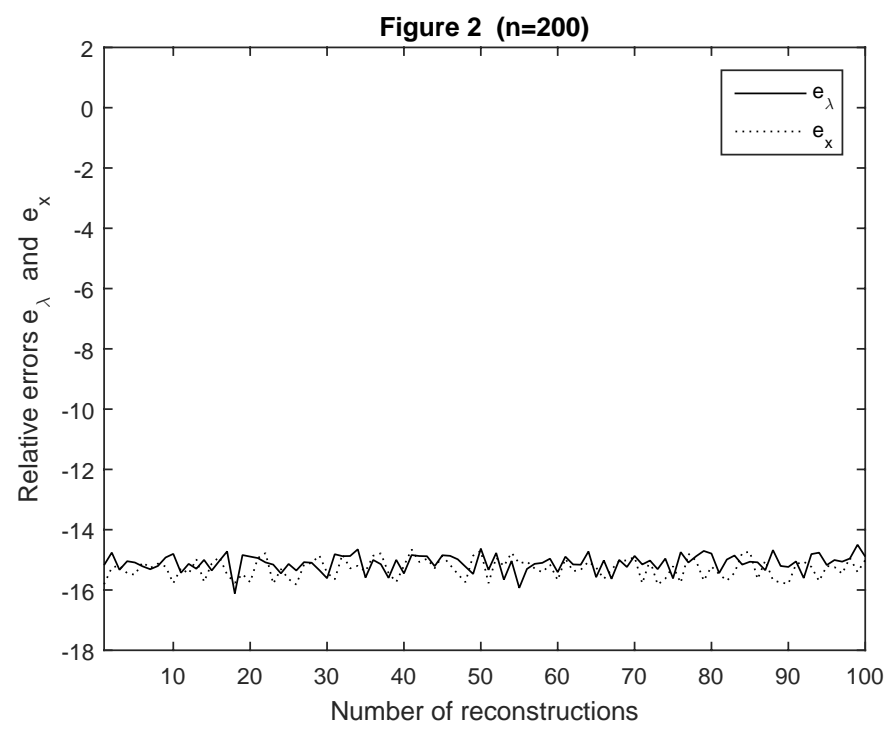

Example 6. Consider the $(n+1)$-dimensional vector $\lambda$, whose entries are real numbers chosen randomly in nondecreasing order, and an $n$ dimensional vector $\mathbf{x}$, satisfying the conditions (3.2) and (3.3) of Theorem 1 , respectively. Figure 2 show the plots of $e_{\lambda}$ and $e_{x}$, defined similarly as in Examples 4 and 5, where we construct 50 nonsymmetric arrowhead matrices $\widetilde{A}$ of order $n=200$.

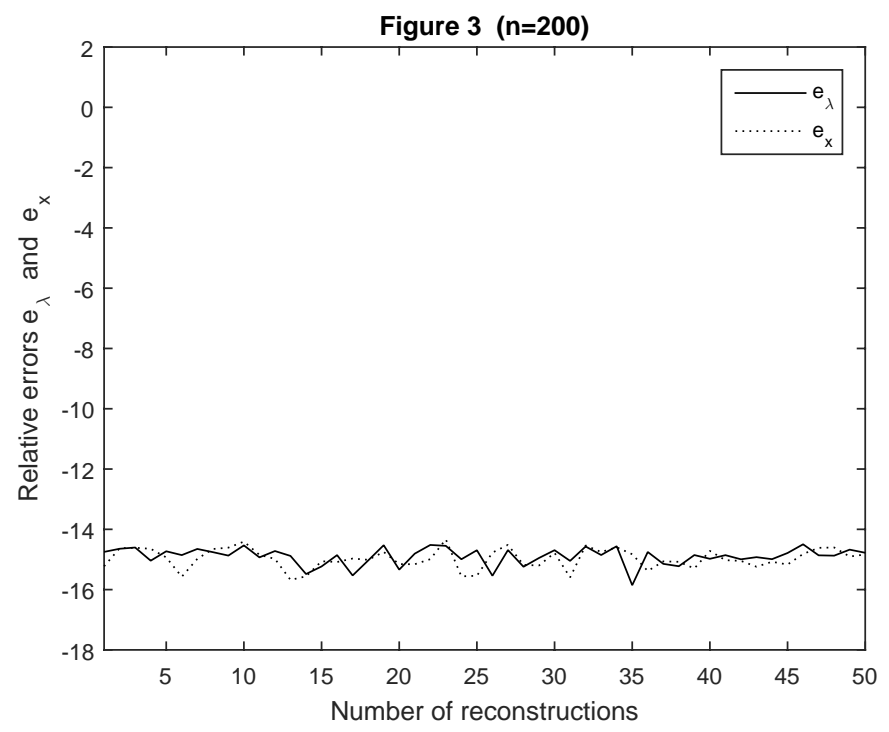




\section{Acknowledgement}

We thank Professor Hector Miranda for suggestions and comments to improve this article.

Supported by Proyecto Mayor de Investigación Científica y Tecnológica UTA 4747-19

\section{References}

[1] M. Bixon and J. Jortner, "Intramolecular radiationless transitions", The Journal of chemical physics, vol. 48, no. 2, pp. 715-726, 1968, doi: $10.1063 / 1.1668703$.

[2] M. Chu and G. Golub, Inverse eigenvalue problems: theory, algorithms, and applications. Oxford: Oxford University Press, 2005, doi: 10.1093/acprof:oso/9780198566649.001.0001.

[3] E. Jessup, "A case against a divide and conquer approach to the nonsymmetric eigenvalue problema", Applied numerical mathematics, vol. 12, no. 5, pp. 403-420, Jul. 1993, doi: 10.1016/01689274(93)90101-V.

[4] V. Higgins and C. Johnson, "Inverse spectral problems for collections of leading principal submatrices of tridiagonal matrices", Linear algebra and its applications, vol. 489, pp. 104-122, Jan. 2016, doi: 10.1016/j.laa.2015.10.004.

[5] A. Nazari and Z. Beiranvand, "The inverse eigenvalue problem for symmetric quasi anti-bidiagonal matrices", Applied mathematics and computation, vol. 217, no. 23, pp. 9526-9531, Aug. 2011, doi: 10.1016/j.amc.2011.03.031.

[6] Z. Li, C. Bu and W. Hui, "Inverse eigenvalue problem for generalized arrow-like matrices", Applied mathematics, vol. 2, no. 12, pp. 1443-1445, Dec. 2011, doi: 10.4236/am.2011.212204.

[7] J. Peng, X. Hu and L. Zhang, "Two inverse eigenvalue problems for a special kind of matrices", Linear algebra and its applications, vol. 416, no. 2-3, pp. 336-347, Jul. 2006, doi: 10.1016/j.laa.2005.11.017.

[8] H. Pickmann, J. Egaña and R. Soto, "Extremal inverse eigenvalue problema for bordered diagonal matrices", Linear algebra and its applications, vol. 427, no. 2-3, pp. 256-271, Dec. 2007, doi: 10.1016/j.laa.2007.07.020.

[9] H. Pickmann, J. Egaña and R. Soto, "Extreme spectra realization by real symmetric tridiagonal and real symmetric arrow matrices", Electronic journal of linear algebra, vol. 22, pp. 780-795, 2011, doi: 10.13001/1081-3810.1474.

[10] H. Pickmann, S. Arela, J. Egaña and R. Soto, "Extreme spectra realization by real nonsymmetric tridiagonal and real nonsymmetric arrow matrices", Mathematical problems in engineering, vol. 2019, Article ID 3459017, Mar. 2019, doi: 10.1155/2019/3459017. 
[11] H. Najafi, S. Edalatpanah and G. Gravvanis, "An efficient method for computing the inverse of arrowhead matrices", Applied mathematics letters, vol. 33, pp. 1-5, Jul. 2014, doi: 10.1016/j.aml.2014.02.010.

[12] L. Shen and B. Suter, "Bounds for eigenvalues of arrowhead matrices and their applications to hub matrices and wireless communications", EURASIP Journal on advances in signal processing, vol. 2009, Article ID 379402, Dec. 2019, doi: 10.1155/2009/379402.

[13] W. Wanicharpichat, "Explicit minimum polynomial, eigenvector, and inverse formula for nonsymmetric arrowhead matrix", International journal of pure and applied mathematics, vol. 108, no. 4, pp. 967-984, 2016, doi: 10.12732/ijpam.v108i4.21. 